\title{
Water Quality Monitoring and Management System for Residents
}

\author{
Anjali Jain, Archika Malhotra, Aayushi Rohilla, Prachi Kaushik
}

\begin{abstract}
This paper presents a Water Quality Monitoring and Management System for the Residents. For the incoming water three water quality parameters- $\mathrm{pH}$, Turbidity, and Temperature will be checked. The proposed system will check the tank level and quality of water supplied and according to the results will decide whether water to be allowed to enter into the cistern. The system notifies the user about water quality and daily water consumption via the IFTTT app, SMS or email alert as data will be uploaded on the Adafruit cloud. The objectives to design this system are to ensure the prevention of contamination of the water supply, untimed water supply and real-time automation of water supply according to the tank level, water quality, and supply.
\end{abstract}

Keywords: Water Management, water quality, real-time data analysis, water Consumption, automate water motor, IFTTT, Adafruit Cloud

\section{INTRODUCTION}

The need for water will never end but according to the reports, water may. So in today's world, it has become a necessity to conserve water to save our future. Fresh and readily available water is the prime need for public health, whether it is used for drinking or household purpose. According to WHO (World Health Organisation) 2.2 billion people consume water without safety procedures.

In many areas household sufferers due to highly dirty and smelly water which is not even suitable for washing and cleaning purposes. This dirty water is due to sewage water entering through broken pipelines. Without any notification, the filthy water gets filled into the water tank of the house and creates the worst scenario for people also leading to serious health issues. This not only wastes the uncontaminated water in tanks but also a huge amount of water is used for cleaning tanks. Due to the untimed water supply, people have to suffer water scarcity for the whole day.

Revised Manuscript Received on December 30, 2019.

* Correspondence Author

Anjali Jain*, Student, Department of Electronics and Communication Engineering, Bhagwan Parshuram Institute of Technology, Rohini (Delhi) India. E-mail: anjalijain1799@gmail.com

Archika Malhotra, Student, Department of Electronics and Communication Engineering, Bhagwan Parshuram Institute of Technology, Rohini (Delhi) India. E-mail: malhotraarchika@gmail.com

Aayushi Rohilla, Student, Department of Electronics and Communication Engineering, Bhagwan Parshuram Institute of Technology, Rohini (Delhi) India. E-mail: aayushirohilla12@gmail.com

Prachi Kaushik, Associate Professor, Department of Electronics and Communication Engineering, Bhagwan Parshuram Institute of Technology, Rohini (Delhi) India. E-mail: prachikaushik@bpitindia.com

(C) The Authors. Published by Blue Eyes Intelligence Engineering and Sciences Publication (BEIESP). This is an open access article under the CC BY-NC-ND license (http://creativecommons.org/licenses/by-nc-nd/4.0/)
To solve this major issue in every household an embedded system is presented that can detect incoming water and check the quality of water. This information will analyze when the motor will be turned on and off automatically according to the water tank level. The status will be notified to the user using IoT.

\section{LITERATURE SURVEY}

Several works have been done for water quality and water management analysis. Brinda Das et .al.[1] presents a Water Quality Monitoring System using IoT which detects the water quality and notifies the values using the GSM module. The system measures various parameters of water and Zigbee Module is used to send the data on the microcontroller and GSM module to send data to a smartphone/PC. An Automatic water level sensor with a controller is presented by Beza Negash Getu et .al. [2] which presents an automated system based on sequential logic implemented using a flip flop. The system detects the level of water in the water tank and controls the water motor. Sajith Saseendran et .al.[3] has come up with an Automated Water Usage Monitoring System to help an individual to keep track of the water bill and to help the government to manage the water supply and modify the billing scheme. The proposed system collects the data through Wi-Fi and track usage and wastage of water at every outlet and sends an alert to the user when water is used in excess. Arjun $\mathrm{K}$ et. al.[4] has proposed a system for Detection of Water Level, Quality, and Leakage using Raspberry Pi with IoT. The system further includes sensors that measure the level of the container, pressure value and quality value and store the value in a database, and notifies the user. Manish Kumar Jha et .al.[5] has presented a solution of SWMS using raspberry pi and Arduino UNO to measure the water quality and to measure the water quantity. This online monitoring system provides data on the Thingspeak cloud and data is compared with the standard quality and also with advised usage.

\section{PROPOSED SYSTEM}

The Water Quality Monitoring and Management System focuses to target the problem of untimed water supply, venturing of contaminated water to enter into tanks, overflowing of tanks and unnoticed daily water consumption. This system will first check the tank level and if the tank level is low then it will check the incoming water supply. Only if the water supply is present then the motor will automatically turn on for a few seconds to check the quality of water. And if the quality of water is desired then the only motor will turn on and anytime if water supply discontinued then the motor will automatically turn off. 
The amount of water flow to the tank will tell the consumption of water from the previous day. This whole process will not be IoT Based as if the internet connection will get lost then the process will terminate, so the process is constructed on an embedded system. Now after the process gets completed the data will be notified to the user using IoT.

The quality of water will be checked based on three parameters- $\mathrm{pH}$, Turbidity and Temperature. $\mathrm{pH}$ will tell about whether the water is acceptable for drinking or not. Turbidity will tell whether the water is even acceptable for any household chores or not. The temperature will tell if the water contains essential elements

Table- I: Water Quality Parameters Standard Value Table

\begin{tabular}{|c|c|c|}
\hline $\begin{array}{l}\text { Water Quality } \\
\text { Parameter }\end{array}$ & $\begin{array}{l}\text { Standard } \\
\text { Value } \\
\text { Range }\end{array}$ \\
\hline $\mathrm{pH}$ & $6.5-8.5$ \\
\hline Turbidity & $0-5$ & NTU \\
\hline Temperature & $0-30$ & $\left({ }^{0} \mathrm{C}\right)$ \\
\hline
\end{tabular}

As the water tank is monitored continuously so overflow will not occur. Due to the water flow check, the untimed water supply problem will be solved. Quality check of incoming water will not let filthy water to enter into the tanks. Daily water consumption, when notified to the user, will alert the user to reduce the wastage of water. With all these parameters water-related problems in-household can be resolved.

\section{A. Block Diagram}

All the sensors are attached to the Arduino Uno. The value of the water tank level will be sent by the ultrasonic sensor to the microcontroller. Flow sensor and water Quality sensors - pH sensor, turbidity sensor, and temperature sensor are connected to the controller. NodeMCU and Arduino UNO connected for serial communication.

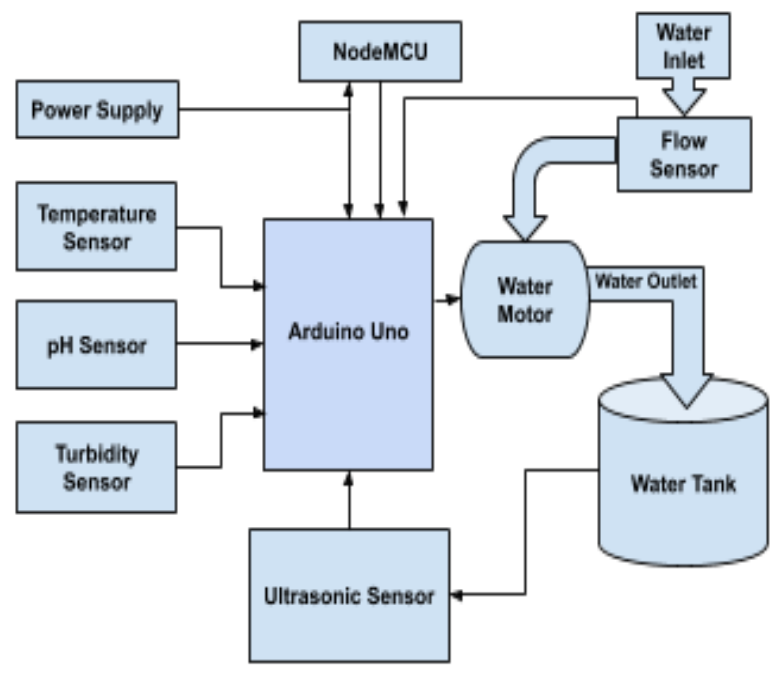

Fig. 1 Block Diagram of the proposed system

\section{B. Algorithm}

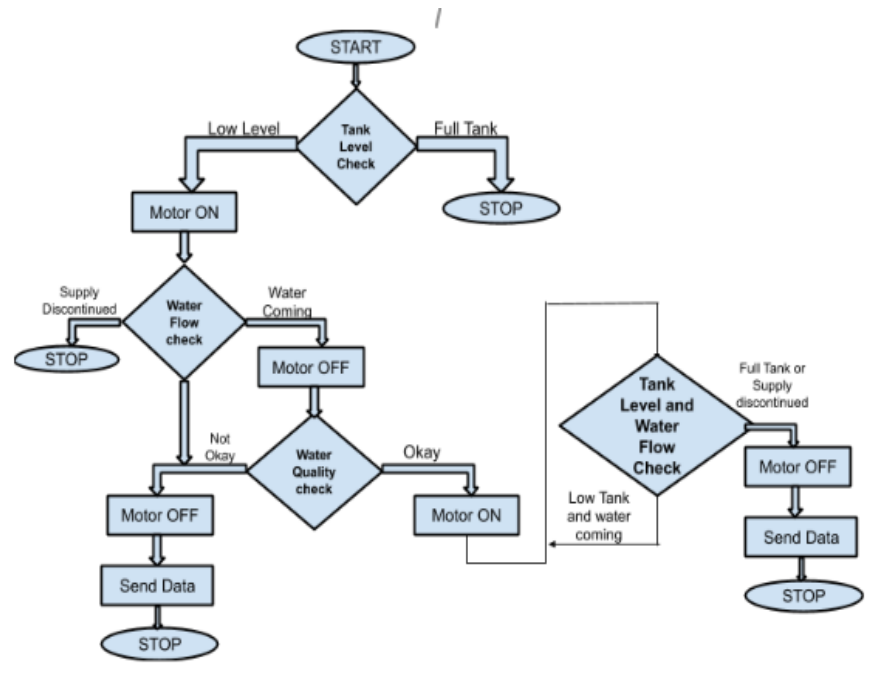

Fig. 2 Flowchart of Water Quality Monitoring and Management System

Algorithm for the proposed system is as follows:

1. First Water Tank Level will be checked and if tank level will be low then the motor will ON or if the tank is full process will stop.

2. Now for low-level water motor will be ON for a few seconds to check whether the water supply is present or not.

3. If supply is not present then the motor will turn OFF. But if the supply will be present then the motor will turn OFF and now the quality of water is checked.

4. If quality is satisfied then the motor will again turn ON and will remain on until the tank is full or water supply is discontinued, after that data will be sent and the process will stop

5. If quality is not satisfied then data will be sent to the cloud and the motor will remain OFF.

\section{IMPLEMENTATION}

\section{A. Hardware}

I. Arduino Uno: Arduino Uno is an ATmega328P based microcontroller board. It is an important component in this system as all the sensors and WiFi module is connected to it. It takes input from the sensor to analyze it and give the command to the motor. It will also send data to $\mathrm{WiFi}$ Module.

II. NodeMCU: It includes firmware that runs on the ESP8266 Wi-Fi SoC and hardware based on the ESP-12 module. It will take data from Microcontroller through serial communication and then send this data to Adafruit IO Cloud.

III. Sensors: Five Sensors are employed in the implementation of the Water Quality Monitoring and Management System. The $\mathrm{pH}$, Turbidity and Temperature sensors are used to measure the quality of water. Flow Sensor is used to detect the flow of water and measure the water consumption. As water flows through the sensor, the rotor rolls. The number of rotations of the rotor gives us the measure of the amount of water that has flown through the flow sensor.

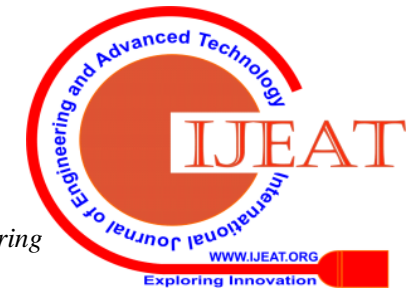


$I V$. Ultrasonic Sensor is used to measure the level of the water tank by sending the ultrasonic signal and then detecting the signal which reflects through the surface of the water.

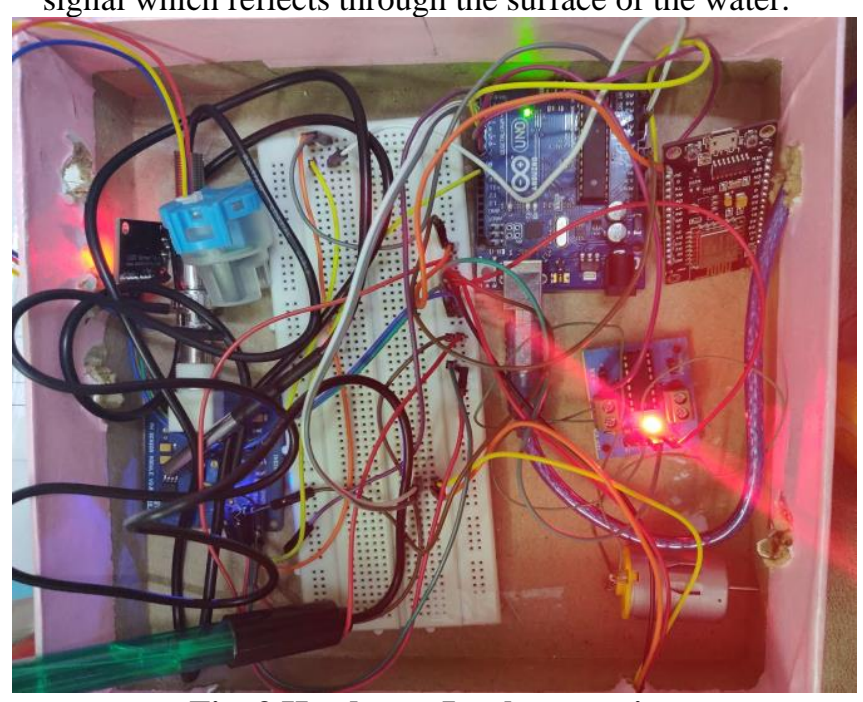

Fig. 3 Hardware Implementation

B. Software

I. Arduino IDE: Arduino IDE is used to program Arduino UNO and NodeMCU. It is open-source software that is mainly used for writing and compiling the code into the Arduino Module. Both Arduino UNO and NodeMCU are connected through serial communication in Arduino IDE.

II. Adafruit IO: Adafruit IO is a cloud service on which different feeds can be created. All the data of the sensors and water consumption value is published to the feeds created in Adafruit IO from NodeMCU through MQTT.

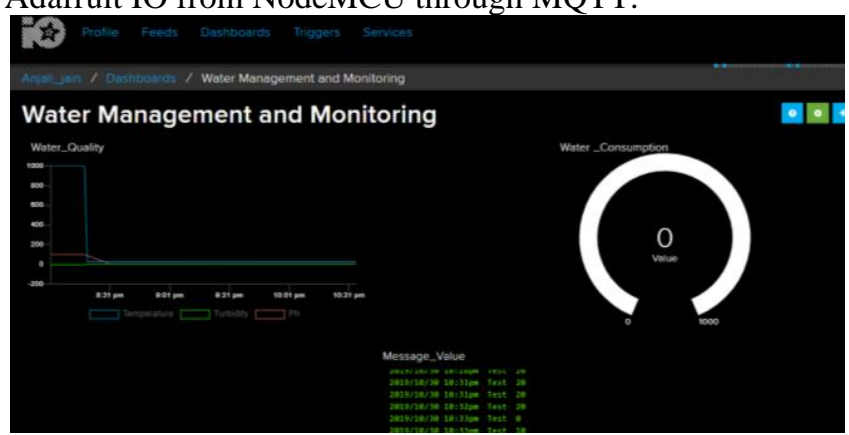

Fig. 4 Water Management and Monitoring System Dashboard in Adafruit IO

III. IFTTT: If This Then That, also known as IFTTT, is a free web-based service in which Applets are created for notifying the user on their Smartphone/PC. Applets created for this system will trigger a notification on the Smartphone based on the data in Adafruit.
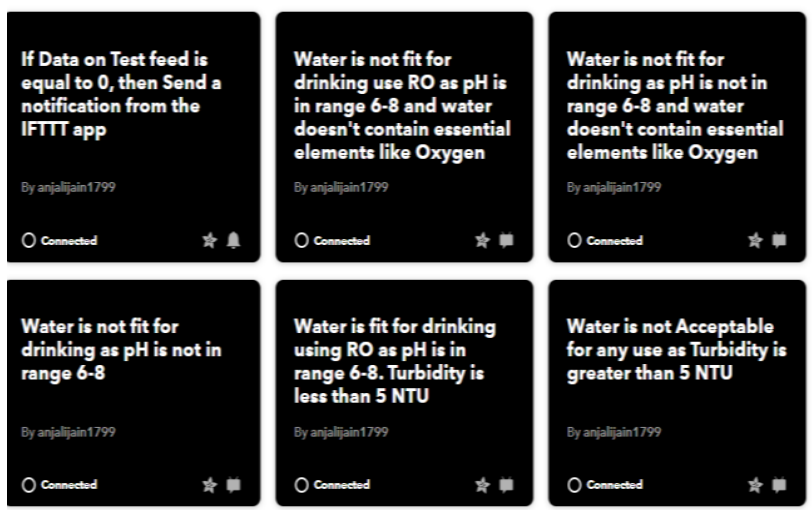

Fig. 5 System Applets in IFTTT

\section{RESULT}

Water Quality Monitoring and Management System is implemented successfully. Fig. 6 shows the value of $\mathrm{pH}$, temperature, turbidity and water consumption in the serial monitor of Arduino IDE that is provided by the embedded system.

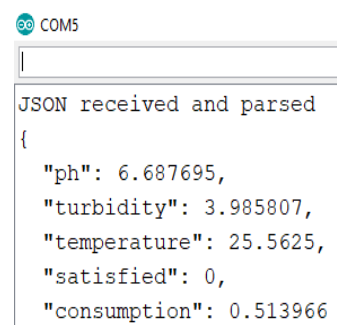

Fig. 6 Serial Monitor

According to these embedded system will decide whether to allow water or not to the resident water tank and also the above data is sent to Adafruit cloud where it is stored is different feeds. Water quality chart is shown in Fig.7 that indicates the values of $\mathrm{pH}$, temperature and turbidity that are sent to feeds in Adafruit IO.

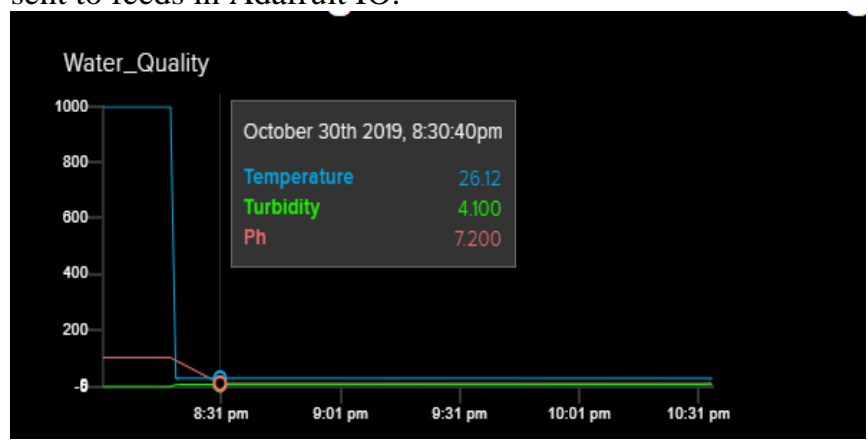

Fig. 7 Water Quality Chart in Adafruit IO

The value of water consumption that is stored to the cloud is shown in Fig.8

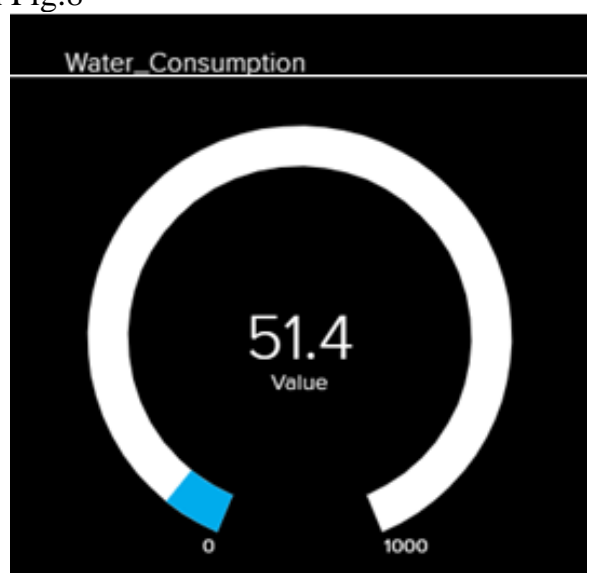

Fig. 8 Water Consumption Feed in Adafruit

Now IFTTT will check the quality and consumption values in Adafruit IO and notifies the user on their smart phone. Fig.9 shows notification sent by IFTTT. Two different notifications will be sent one for the water consumption value and one for notifying about the water acceptance level.

\section{Published By:}




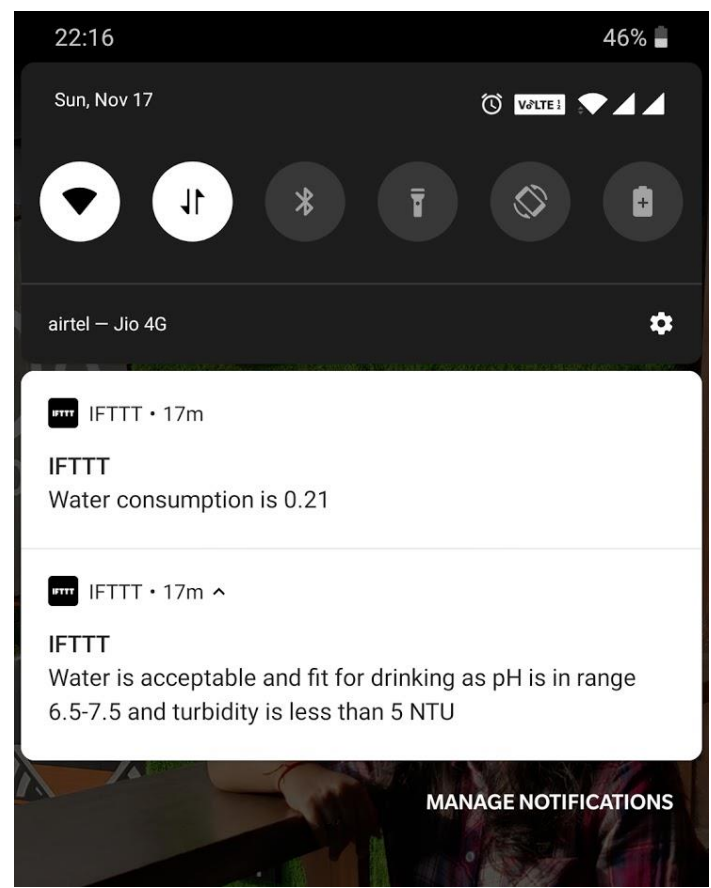

Fig. 9 IFTTT App Notifications

\section{CONCLUSION}

This Water Quality Monitoring and Management System is providing the desired and efficient value of the level of the cistern, incoming water supply and quality of water and controlling the motor or solenoid valve according to the data. This embedded system is running automatically and does not need any human interference. It will run even without the WiFi connection. The WiFi will be needed only for transmitting data to the user interface else the system can act as a Stand-Alone Device. The system

\section{FUTURE SCOPE}

A user interface can be designed and control from that interface can be given to the user so that the user can decide whether to discard the water supply or not. An integrated sensor system can be developed which can monitor all the parameters of water to analyze the quality.

\section{REFERENCES}

1. Brinda Das and P.C. Jain, "Real-Time Water Quality Monitoring System using the Internet of Things", published in 2017 IEEE International Conference on Computer, Communications and Electronics (Comptelix), Accession Number: 17085193, 01-02 July 2017

2. Beza Negash Getu and Hussain A. Attia, "Automatic Water Level Sensor and Controller System”, published in 2016 5th Internationa Conference on Electronic Devices, Systems and Applications (ICEDSA), Accession Number: 16616146, 19 January 2017

3. Sajith Saseendran and V. Nithya, "Automated Water Usage Monitoring System”, published in 2016 IEEE International Conference on Communication and Signal Processing, Accession Number: 16488735, April 6-8, 2016.

4. Arjun K, Latha C A, and Prithviraj, "Detection of Water Level, Quality and Leakage using Raspberry Pi with Internet of Things", published in International Research Journal of Engineering and Technology (IRJET), Volume 04 Issue 06, June 2017, pp 2875-2880.

5. Manish Kumar Jha, Rajni Kumari Sah, M. S. Rashmitha, Rupam Sinha, B. Sujatha, and K.V. Suma, "Smart Water Monitoring System for Real-Time Water Quality and Usage Monitoring”, published in 2018 International Conference on Inventive Research in Computing Applications (ICIRCA), Accession Number: 18358016, 03 January 2019.
6. Taufik Ibnu Salim, Hilman S. Alam and Rian P. Pratama, "Portable and online water quality monitoring system using wireless sensor network", published in 2nd International conference IEEE ICACOMIT 2017, Accession number: 17487078, January 2018.

7. Shuang-Hua Yang, Xi Chen, Xiaomin Chen, Lili Yang, Baichong Chao, Jiangtao Cao, “A case study of Internet of things: A wireless household water consumption monitoring system", published in 2015 IEEE 2nd World Forum on Internet of Things (WF-IoT), Accession Number: 15729217, December 2015

\section{AUTHORS PROFILE}
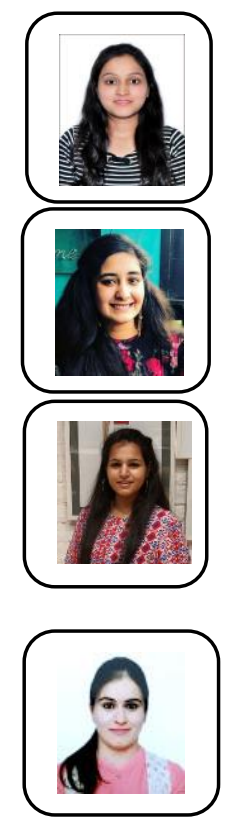
Engineering. Her specialization is in Wireless Communication. Email: prachikaushik@bpitindia.com

Anjali Jain, is currently pursuing her Bachelor's degree in Electronics and Communication from Bhagwan Parshuram Institute of Technology, Rohini, Delhi, India. She is a student member of IEEE.

Email: anjalijain1799@gmail.com

Archika Malhotra, is currently pursuing her Bachelor's degree in Electronics and Communication from Bhagwan Parshuram Institute of Technology, Rohini, Delhi, India. She is a student member of IEEE.

Email: malhotraarchika@gmail.com

Aayushi Rohilla, is currently pursuing her Bachelor's degree in Electronics and Communication from Bhagwan Parshuram Institute of Technology, Rohini, Delhi, India. She is a student member of IEEE.

Email: aayushirohilla12@gmail.com

Prachi Kaushik, is an Associate Professor at Bhagwan Parshuram Institute of Technology, Rohini, Delhi, India She has received her Bachelor's degree from Guru Jamshedpur University of Science and Technology in Biomedical Engineering and Master's from Maharshi Dayanand Universiy in Electronics and Communication 